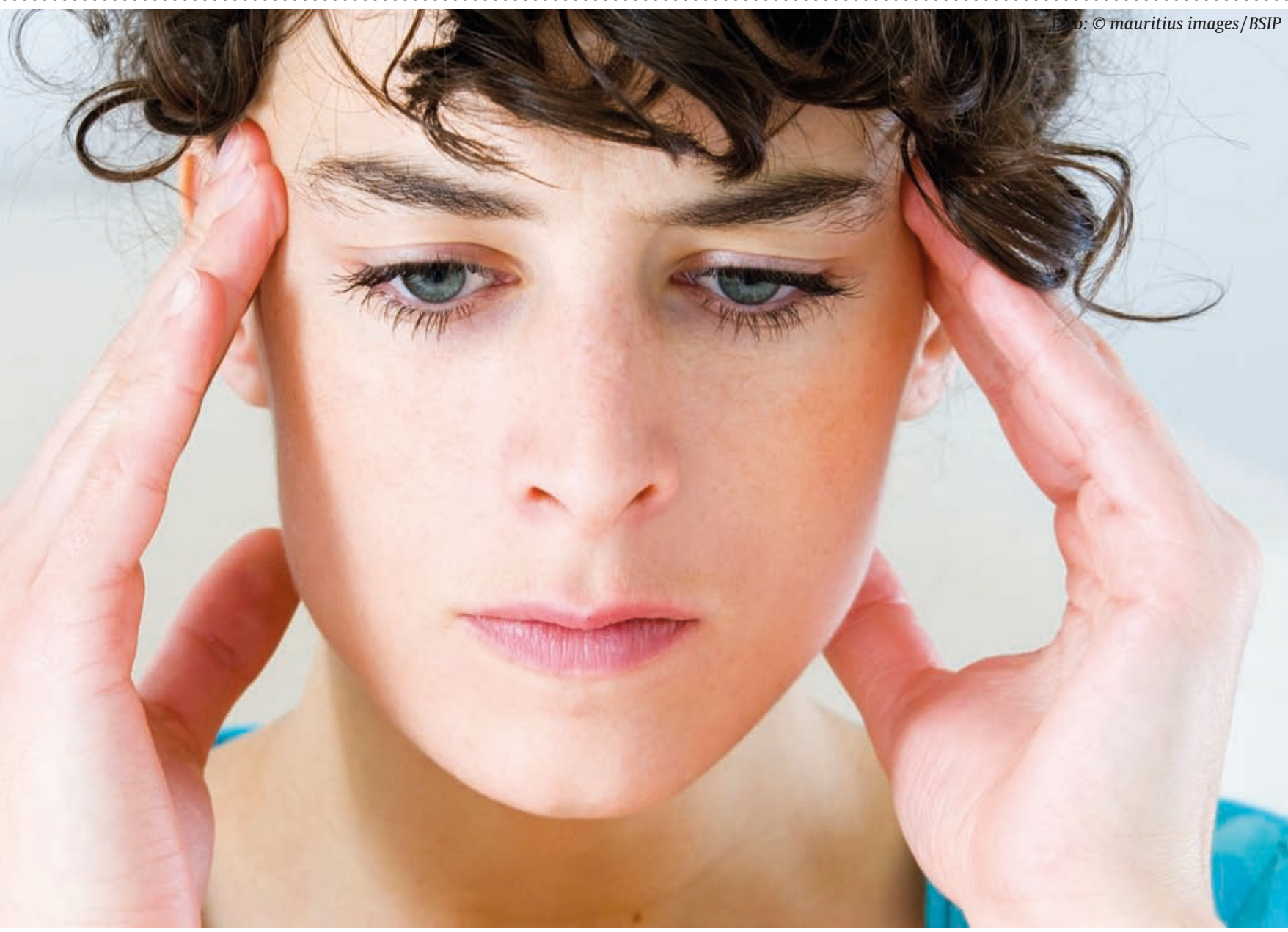

\title{
In der Schraubzwinge
}

\section{Der URSACHE des Kopfschmerzes auf die Schliche kommen}

\section{Kerstin Holdt}

ALS OB der Kopf in einer Schraubzwinge steckt. So beschrieb neulich eine Patientin (35) ihre Kopfschmerzen, unter denen sie seit Wochen immer wieder litt. Sie waren mal mehr und mal weniger stark ausgeprägt, manchmal nur für eine oder zwei Stunden vorhanden; einmal quälten sie die Frau fast zwei Tage am Stück. Wenn die Kopfschmerzen auftraten, störten Geräusche oder Licht. Einmal ging die Patientin trotz der Schmerzen joggen, danach waren sie weg.

Sie nahm an, der viele Stress bzw. ihre ungünstige Körperhaltung vor dem PC, die häufig zu Nackenverspannung führt, seien Ursache für die Schmerzen. Doch weil sie wieder und wieder auftraten, sorgte sich die Patientin, die Kopfschmerzen könnten Symptom von etwas Ernstem sein.

Als mir die Frau in der Praxis gegenüber saß und die Symptome schilderte, konnte ich ihr die Angst ein wenig nehmen. Denn ihre Symptome deuteten auf den sogenannten Kopfschmerz vom Spannungstyp bzw. Spannungskopfschmerz hin.
Die von ihr beschriebene Schmerzcharakteristik ist für diese Kopfschmerzform typisch: milde bis moderate Schmerzen (beidseitig) mit dumpfem, drückendem Charakter und einer Betonung im Stirn- und Hinterkopfbereich. Die unterschiedliche Schmerzstärke und die Dauer - der Spannungskopfschmerz kann gelegentlich bis wöchentlich, für 30 Minuten bis zu sieben Tage am Stück auftreten -, keine Übelkeit, gelegentliche Lichtund Geräuschempfindlichkeit (nie gleichzeitig) sowie Besserung durch Sport sind weitere klassische Zeichen. Insgesamt

\section{Was schmerzt im Kopf?}

Wenn der Kopf schmerzt, schmerzen nicht das Gehirn oder der Schädelknochen, sondern es sind die Strukturen, die vom N. trigeminus oder der Hinterwurzel des ersten zervikalen Spinalnervs innerviert werden. Die größeren zerebralen Gefäße, die venösen Sinus, die spinalen Gefäße sowie die Dura mater zählen dazu. 
leiden $42 \%$ der Erwachsenen an einem sporadischen oder häufigen zeitweisen und $3 \%$ an einem chronischen Spannungskopfschmerz. Entgegen der landläufigen Meinung sind Männer so häufig wie Frauen betroffen.

\section{Primärer Kopfschmerz ist häufig die Diagnose}

Neben Migräne und Clusterkopfschmerz sowie Kopfschmerzen bei körperlicher oder sexueller Anstrengung zählt der Spannungskopfschmerz zu den primären Kopfschmerzformen. Sie machen $90 \%$ aller Kopfschmerzen aus - primär, weil der Schmerz nicht Symptom ist, sondern selbst die Erkrankung. Was den primären Kopfschmerz auslöst, ist auch heute noch weitgehend unklar. Psychische Faktoren scheinen für die Ausprägung und den Verlauf bedeutend. Die schweren Formen mit häufigen, lang anhaltenden und heftigen Schmerzen sind Studien zufolge jedoch oft neurologisch bedingt. Die genaue Ursache kennt man noch nicht. Wissenschaftler gehen davon aus, dass Veränderungen in den schmerzverarbeitenden Bereichen des Gehirns eine Rolle spielen.

Auslöser für den Spannungskopfschmerz, die häufigste primäre Kopfschmerzform, sind oft körperlicher oder psychischer Stress sowie muskuläre Fehlbelastungen. Sie führen dazu, dass muskuläre Triggerpunkte schmerzempfindlicher werden, was wahrscheinlich zur Folge hat, dass schmerzverarbeitende Strukturen sensibilisiert und die endogenen antinozizeptiven Einflüsse gedämpft werden. Das kann auf Dauer in einen Teufelskreis und die Chronifizierung führen.

Sekundäre Kopfschmerzen machen die übrigen 10\% aller Kopfschmerzfälle aus, der Kopfschmerz ist dann Symptom und nicht Ursache. Er tritt in engem zeitlichen Zusammenhang mit

\section{- INFORMATION}

\section{Spannungskopfschmerz: Unterteilung in episodische und chronische Verlaufsform}

Gemäß der Internationalen Klassifikation von Kopfschmerzen (ICHD-II) unterscheidet man zwischen einer episodischen und einer chronischen Verlaufsform:

- Der episodische Spannungskopfschmerz wird weiter unterteilt in den sporadischen Subtyp mit weniger als ein Tag pro Monat und einen Subtyp mit häufigeren Attacken (max. 10 Tage pro Monat). Der sporadische Subtyp ist weniger bedeutend, es suchen deswegen kaum Patienten die Praxis auf. Der Subtyp mit häufigeren Attacken ist beeinträchtigend und damit behandlungsrelevant.

- Die chronische Verlaufsform (3\%) wird diagnostiziert, wenn der Spannungskopfschmerz mindestens drei Monate an mehr als 15 Tagen / Monat auftritt. Oft treten die Kopfschmerzen täglich auf, und häufig leiden die Betroffenen gleichzeitig unter psychischen Störungen, insbesondere einer Depression.

\section{KURZ GEFASST}



einer anderen Erkrankung auf, beispielsweise Tumoren. Aber auch Infektionen des Gehirns bzw. der Hirnhäute und vieles andere können Ursachen sein (s. Tabelle 1).

\section{Vom Schlimmsten ausgehen}

Auch wenn alle Symptome auf den Spannungskopfschmerz hindeuten: Notfälle oder Infektionskrankheiten müssen ausgeschlossen werden, wenn ein Patient mit Kopfschmerzen in die Praxis kommt. Daher ist neben aufmerksamem Zuhören, wenn der Patient seine Beschwerden schildert, immer eine ausführliche Anamnese und körperliche Untersuchung notwendig.

Zunächst erfragt man den Schmerzcharakter und eventuelle Schmerzausstrahlungen, Lokalisation, Dauer und Frequenz der Kopfschmerzen sowie eventuelle Begleitsymptome wie Lichtempfindlichkeit, Übelkeit, Erbrechen, Sehstörungen etc. Zudem sollte man auf mögliche Hirndruckzeichen achten. Man misst immer Blutdruck, Puls und Temperatur. Die Pupillen sind auf eine eventuelle Anisokorie hin zu kontrollieren.

Die körperliche Untersuchung umfasst auch neurologische Tests, insbesondere der Hirnnerven und Reflexe, ebenso wie die Beweglichkeit der Wirbelsäule und der Extremitäten, u. a. Romberg-Test und Unterberger-Tretversuch.

Bei der Anamnese und Untersuchung führen die Antworten bzw. Befunde dann zu weiteren Schritten in der Differenzialdiagnostik. Diese kann unter Umständen sehr breit gefächert

- INFORMATION
Hirndruckzeichen
- Kopfschmerzen
- Sehstörungen, Pupillenveränderungen
- anderweitig nicht begründbare Übelkeit/ Erbre-
chen im Schwall
- Hypertonie
- Bradykardie (Druckpuls)
- Wesensveränderungen, Vigilanzstörungen
- Meningismus


ausfallen. In diesem Beitrag auf alle Möglichkeiten einzugehen ist nicht möglich. Ein Beispiel soll das Vorgehen exemplarisch verdeutlichen:

Ein Patient gibt auf die Frage nach der Schmerzlokalisation an, dass es sich um einen Halbseitenkopfschmerz handelt. Ist bei der Untersuchung der Pupillen eine lichtstarre einseitige Mydriasis zu sehen, könnte die Ursache ein akutes Glaukom sein. Ein Notfall! Um den Verdacht zu erhärten bzw. ein Glau-

- TABELLE 1

\section{Sekundäre Kopfschmerzen und ihre Ursachen}

\begin{tabular}{|c|c|}
\hline $\begin{array}{l}\text { Orthopädisch / } \\
\text { osteopathisch }\end{array}$ & $\begin{array}{l}\text { Schleudertrauma } \\
\text { Verspannungen der Hals-Nacken-Muskulatur } \\
\text { Degenerative Veränderungen der HWS } \\
\text { Skoliose } \\
\text { HWS-Syndrom } \\
\text { myofasziale, zirkulatorische und viszerale Dysfunktionen } \\
\text { Dysgnathien (unphysiologische Verschiebung der Zahn- } \\
\text { reihen) } \\
\text { CMD (Craniomandibuläre Dysfunktion) }\end{array}$ \\
\hline HNO & $\begin{array}{l}\text { Sinusitis } \\
\text { Rhinitis } \\
\text { Otitis media (externa und interna) }\end{array}$ \\
\hline Internistisch & $\begin{array}{l}\text { Hypertonie (renal, endokrin, kardiovaskulär bedingt) } \\
\text { Blutdruckschwankungen (Hypotonie) } \\
\text { Hormonelle Störungen (Hypothyreose) } \\
\text { Infektionskrankheiten (fast alle, die mit Fieber einher- } \\
\text { gehen) } \\
\text { Allergien } \\
\text { Übertragener Schmerz (referred pain) }\end{array}$ \\
\hline Neurologisch & $\begin{array}{l}\text { Erhöhung des Hirndrucks } \\
\text { Intrakranielle Raumforderung (Tumor, Ödem, Aneurysma, } \\
\text { Blutungen) } \\
\text { Epilepsie } \\
\text { SHT (Commotio, Contusio inkl. Schädelbasisbruch, } \\
\text { Compressio cerebri) } \\
\text { Apoplex (TIA, PRIND, Insult) } \\
\text { Meningitis/FMSE (IfSG beachten!) } \\
\text { Herpes Zoster (IfSG beachten!) } \\
\text { Zerebrale Gefäßanomalien } \\
\text { Neuralgien (Trigeminus, Facialis) } \\
\text { Arteriitis temporalis/M. Horton }\end{array}$ \\
\hline Psychogen & $\begin{array}{l}\text { Stress } \\
\text { Klima } \\
\text { Schlafmangel }\end{array}$ \\
\hline $\begin{array}{l}\text { Ophthalmolo- } \\
\text { gisch }\end{array}$ & $\begin{array}{l}\text { Sehstörungen } \\
\text { Akutes Glaukom }\end{array}$ \\
\hline Noxen & $\begin{array}{l}\text { Medikamente } \\
\text { Nahrungsmittel } \\
\text { Lärm } \\
\text { Alkohol }\end{array}$ \\
\hline
\end{tabular}

kom auszuschließen, palpiert man als nächstes vorsichtig den Augapfel (Bulbus oculi). Bei einem Glaukom wäre dieser hart. Man fragt dann weiter nach Farbensehen. Ist auch dieses Symptom vorhanden, ist der Verdacht bestätigt, und der Patient muss sofort an einen Augenarzt bzw. in die Augenklinik verwiesen werden. Ist die Pupille lichtträge, der Bulbus nicht steinhart und imponieren schmerzhafte Akkommodationsstörungen, könnte eine einfache Iritis vorliegen. Auch dann müsste der Patient an einen Augenarzt überwiesen werden. Eine Anisokorie kann ein Hinweis auf intrazerebrale Blutungen sein, ebenfalls ein Notfall! Es finden sich dann zusätzlich oft eine ipsilaterale Mydriasis und kontralaterale Hemiparese.

\section{Merke: Pupillendifferenz und Hemiparese sind immer ernst zu nehmen und ein Notfall. Es sei denn, sie sind dem Patienten bekannt.}

Hat der Patient mit den Halbseitenkopfschmerzen Fieber, sollte man an eine FSME (Frühsommer-Meningoenzephalitis) oder Meningokokkenmeningitis denken. Neben weiteren anamnestischen Fragen nach Zeckenstich, allgemeinen Infektionszeichen, Symptomen der Meningitis bzw. FSME untersucht man den Patienten auf Nackensteifigkeit, ein klassisches Meningismus-Zeichen. Ist es vorhanden, muss der Patient sofort in eine Klinik überwiesen werden.

Ist der Halbseitenkopfschmerz nicht von Fieber oder einer Anisokorie begleitet, imponiert aber eine geschlängelte, schmerzhafte und gestaute Arteria temporalis superficialis, könnten eine Arteriitis temporalis, aber auch eine Herpes-Zoster-Infektion des Nervus ophthalmicus (erster Ast des Trigeminusnervs) die Ursache sein. In beiden Fällen können die Patienten erblinden, weshalb dies ebenfalls eine Notfallsituation ist. Zudem: Herpes Zoster darf ein Heilpraktiker gemäß Infektionsschutzgesetz (IfSG) nicht behandeln.

\section{Die Suche nach der Nadel im Heuhaufen}

Wenn Notfälle und Infektionskrankheiten ausgeschlossen sind, sollte nach weiteren Ursachen gesucht werden (weiterführende Anamnese). Es erfordert mitunter viel Geduld, den Grund für den Kopfschmerz als Symptom zu differenzieren. Denn die Ursachen können sehr verschieden sein (s. Tabelle 1). Einige Beispiele für die Differenzierung:

- Schmerzen/Knacken bei Kieferöffnung oder eine fehlerhafte Kieferokklusion könnten Hinweise auf eine Craniomandibuläre Dysfunktion sein.

- Nahrungsmittelunverträglichkeiten können ebenfalls Kopfschmerzen auslösen.

- Die trigeminalen Nervenaustritte sollte man palpatorisch auf Druckschmerzhaftigkeit prüfen. Sie sind Triggerpunkte und können eine Trigeminusneuralgie auslösen.

- Die Beweglichkeit der HWS sollte man testen, eine Einschränkung kann ein Hinweis auf ein HWS-Syndrom sein, ebenso die Kalotte auf Klopf- und Druckschmerz. 
- Eine Blutlaboruntersuchung könnte im nächsten Schritt durchgeführt werden, um einen Verdacht, der sich aus der Anamnese ergeben hat, zu stützen. Vielleicht ist der Kopfschmerz hormonell bedingt (Hypothyreose)? Eine Bestimmung von TSH, T3 und T4 würde das bestätigen.

- Bei Verdacht auf ein Trauma sind ggf. bildgebende Verfahren und eine Überweisung zu einem Facharzt zur weiteren Abklärung notwendig (HWS-Röntgen: knöcherne Destruktionen bei frischem Trauma/knöcherne Verhältnisse der HWS; HWS-Gefügeschäden; Spondylolisthesis, ligamentäre Läsionen etc).

\section{Bei Spannungskopfschmerz ist die perikranielle Muskulatur druckschmerzhaft}

Lassen sich sekundäre Kopfschmerzformen sowie andere primäre ausschließen, und deuten die übrigen Symptome auf Spannungskopfschmerz hin, testet man die Druckschmerzhaftigkeit der perikraniellen Muskulatur. Dazu zählen M. frontalis, M. temporalis, M. masseter, Mm. pterygoidei, M. sternocleidomastoideus, M. splenius und M. trapezius. Man prüft mit kleinen, kreisenden Bewegungen unter festem Druck, ob und wie schmerzempfindlich sie sind. Bei Patienten mit Spannungskopfschmerzen ist die Druckschmerzhaftigkeit erhöht. Hinzu kommt: Je häufiger und stärker die Kopfschmerzen sind, desto druckschmerzhafter sind diese Punkte. Während der Kopfschmerzphase ist die Schmerzempfindlichkeit nochmals erhöht.

Dieser Artikel ist online zu finden:

http://dx.doi.org/10.1055/s-0036-1584254

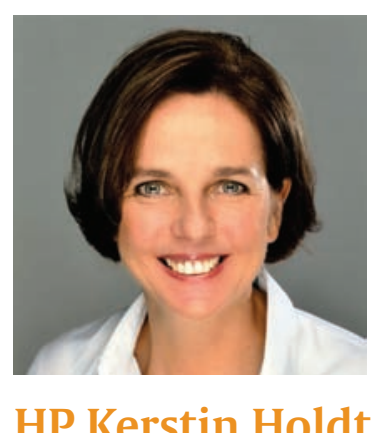

Viersener Str. 10, 50733 Köln

E-Mail: info@naturheilpraxis-holdt.de

Kerstin Holdt ist seit 2009 Heilpraktikerin und seit 2012 DO.CN. Ihre Schwerpunkte in der Praxis sind Chiropraktik, Osteopathie, Neuraltherapie, Blutegelbehandlung, Ausleitungsverfahren, Eigenblutbehandlung, Infusionstherapie sowie minimalinvasive medizinische Ästhetik. 